\section{Ciclo de vida domiciliar, ciclo do lote e mudança no uso da terra na Amazônia brasileira: revisão crítica da literatura}

\author{
Gilvan Ramalho Guedes* \\ Bernardo Lanza Queiroz** \\ Alisson Flávio Barbieri*** \\ Leah Karin VanWey****
}

\section{Introdução}

Diante do influxo de migrantes entre 1965 e 1990 para a Amazônia brasileira, cresceu a preocupação por parte de ecólogos, ambientalistas e cientistas sociais sobre o futuro da Floresta Amazônica devido à divulgada associação entre expansão dos colonos, degradação ambiental e violência social (SIMMONS et al., 2007; FOX; BROWN, 1988). Assim, emergiu de forma clara a discussão sobre a colonização de fronteiras e os problemas sociais e ambientais relacionados ao modelo de desenvolvimento adotado - uma combinação de ocupação do interior da Amazônia com baixa participação do Estado na criação de infraestrutura e canais de financiamento da produção (BROWDER; GODFREY, 1997).

Essa discussão remete às "teorias de fronteira" - nome herdado do trabalho original de Turner (1920) -, entre as quais a teoria do ciclo de vida domiciliar tem sido a mais influente para explicar a evolução das fronteiras agrícolas, em especial na Amazônia, a despeito das fracas evidências comprovando sua validade empírica (VANWEY;
D’ANTONA; BRONDÍZIO, 2007; WALKER et al., 2002). Essa teoria é baseada nos escritos originais de Chayanov (THORNER; KERBLAY; SMITH, 1986), os quais sugerem que o domicílio rural adapta suas estratégias de sobrevivência e de uso da terra às suas demandas (necessidades de consumo) e à capacidade de ofertar trabalho domiciliar, à medida que envelhece. Essa mudança na composição demográfica no interior do domicílio rural, ao longo do tempo, é denominada pela literatura como ciclo de vida domiciliar (PERZ, 2001) e aquela referente ao tempo de residência no/ existência do lote é chamada de ciclo do lote (BARBIERI et al., 2005).

Modelos mais recentes (CALDAS et al., 2007; WALKER, 2004) incorporam uma função de produção domiciliar ao modelo de subsistência de Chayanov, de modo a considerar a influência dos mercados de mão de obra, produção e crédito sobre o gerenciamento da terra.

A adaptação dos modelos de tomada de decisão domiciliar sobre as estratégias de uso do solo reflete um cenário mais complexo das fronteiras agrícolas amazônicas à medida que elas evoluem no tempo e no espaço (SUMMERS, 2008). Diferentemente do ambiente encontrado pelos migrantes nos anos 1970 e 1980, as fronteiras desenvolveram relações diretas e indiretas com os crescentes mercados urbanos, modificando as preferências e oportunidades dos domicílios rurais (SHERBININ et al., 2008).

Com a abertura de estradas, aumento da urbanização e mudança no valor da terra e das commodities, os domicílios passaram a responder crescentemente aos incentivos externos de forma multifásica, ajustando sua fecundidade, mas principalmente utilizando a migração dos membros do domicílio (BARBIERI; CARR; BILSBORROW, 2009; VANWEY; GUEDES; D'ANTONA, 2008) e a diversificação entre setores da economia (MURPHY, 2001) como os principais me-

\footnotetext{
* Pesquisador associado do Environmental Change Initiative / Brown University e do Anthropological Center for Training and Research on Global Environmental Change / Indiana University at Bloomington.

** Professor adjunto nível II do Cedeplar/UFMG.

*** Professor adjunto nível II do Cedeplar/UFMG.

**** Professor associado de Sociology / Brown University; e membro do corpo docente do Environmental Change Initiative / Brown University.
} 
canismos de sobrevivência nesse cenário pós-fronteira. $\mathrm{O}$ aumento da participação do domicílio em atividades não-agrícolas e a crescente venda da produção agropecuária aos mercados locais, regionais e nacionais são considerados, pela literatura, indicadores da crescente capacidade de integração das fronteiras com os mercados.

Por meio de uma revisão sistemática da literatura sobre dinâmica demográfica e uso da terra em fronteiras agrícolas da Amazônia, encontraram-se evidências de que a abordagem do ciclo de vida domiciliar tem recebido pouco suporte empírico, especialmente em relação aos sistemas e classes de gerenciamento da terra. A dinâmica demográfica domiciliar tem maior influência somente em relação ao desmatamento, especialmente em fronteiras agrícolas mais recentes. Por outro lado, os resultados empíricos aqui reunidos sugerem que o ciclo do lote, aproximado pelo tempo de residência no domicílio rural, e a integração aos mercados de produção e venda predominam como uma consequência da consolidação das fronteiras e da mudança no capital humano e natural dos pequenos agricultores, ao longo do desenvolvimento da fronteira.

\section{Metodologia}

Neste trabalho, utilizou-se, como procedimento metodológico, uma revisão sistemática ${ }^{1}$ da literatura sobre mudança no uso e na cobertura da terra em fronteiras agrícolas no nível do domicílio. Ao todo, foram revisados 30 artigos publicados em periódicos internacionais, obtidos por meio de busca realizada entre janeiro e março de 2010. Foram selecionados artigos nas seguintes bases de dados: JStor, Elvisier, Scielo, UCAT, banco de teses do Cedeplar e biblioteca do Cedeplar. Como critérios de sistematização, selecionaram-se os artigos que: apresentavam alguma estratégia em- pírica, além da contribuição teórico/metodológica; tivessem como unidade privilegiada de análise o domicílio rural e/ou o seu lote; fossem aplicados a alguma fronteira agrícola da Amazônia (incluindo sua extensão não-brasileira); e incorporassem ao menos uma das dimensões analisadas (ciclo de vida domiciliar - CVD, ciclo do lote - CL e integração com os mercados de produção e venda - IM).

A opção por selecionar artigos com foco em fronteiras agrícolas justifica-se pelo fato de a revisão sistematizar a influência do CVD, CL e IM especificamente em fronteiras agrícolas, e não em outros tipos de fronteiras, já que é exatamente naquelas que os efeitos do CVD e CL são considerados mais importantes pelos arcabouços teóricos (THORNER; KERBLAY; SMITH, 1986; ELLIS, 1993). O critério de ter teste empírico de pelo menos uma das dimensões (CVD, CL ou IM) justifica-se pelo interesse em ver as evidências empíricas, que validem os arcabouços teóricos, argumentarem sobre a direção de causalidade entre as três dimensões e a mudança no uso da terra.

Os indicadores de CVD variam de artigo para artigo, sendo utilizados para localizar o domicílio em seus vários estágios do ciclo de vida. Assim, as seguintes variáveis apareceram como localizadores do estágio do ciclo de vida nos 30 artigos revisados: número de adultos; número de dependentes; número de homens adultos; número de mulheres adultas; número de crianças; número de idosos; razão de dependência demográfica domiciliar (idosos + crianças / adultos); e idade do chefe do domicílio.

Os indicadores de CL representam a experiência do domicílio com o ambiente biofísico e, em todos os artigos, são aproximados pelo tempo de residência no lote. Por fim, os indicadores de IM procuram mensurar as barreiras de integração dos domicílios aos mercados. Nos artigos revisados, dois indicadores foram empregados: distância ao

\footnotetext{
1 O procedimento procura se enquadrar no esquema de revisão sistemática da literatura, conforme discutido por Rother (2007). Ou seja, partimos de uma pergunta de pesquisa e, com base nesta pergunta, buscamos identificar artigos que tratassem de responder um de seus elementos, pelo menos (no nosso caso, a influência de pelo menos o ciclo de vida, ciclo do lote e/ou integração com o mercado sobre o uso do solo). Sistematizamos, a seguir, os resultados encontrados por esses artigos em relação ao uso do solo, com o objetivo de encontrar um padrão de influência comum.
} 
mercado (distância euclidiana ou distância de rede, partindo do centroide do lote até o centroide do mercado urbano/local mais próximo e distância/tempo gasto do lote até a rodovia principal); e indicador de acessibilidade ao lote durante as estações chuvosas.

Com base nesses indicadores, sugere-se a análise de seus efeitos em duas direções: positivo e negativo. Com o objetivo de apresentar uma tabela-resumo (Quadro 1), foram sumarizados todos os efeitos de cada um dos indicadores do CVD, CL e IM, de modo a representar apenas uma direção do efeito sobre a variável dependente analisada. Por exemplo, em relação aos indicadores de IM (acessibilidade e distância ao mercado), tem-se um efeito positivo se a melhor acessibilidade e maior proximidade com os mercados tendem a aumentar o uso comercial do lote ou elevar a renda agrícola domiciliar. Paralelamente, efeitos positivos do CVD e CL podem ser entendidos como domicílios com menor razão de dependência e maior tempo de residência, sendo mais voltados para culturas comerciais e com menores áreas em mata primária.

\section{Resultados}

O Quadro 1 reúne todos os resultados obtidos na revisão sistemática dos 30 artigos sobre dinâmica demográfica e uso da terra nas fronteiras agrícolas da Amazônia. São listados os autores e o ano da publicação, a região geográfica (fronteira agrícola) onde os dados foram coletados, a variável dependente (cobertura ou classe de uso da terra ou indicador de renda/bens) e os três principais vetores considerados indutores da mudança no uso da terra: ciclo de vida domiciliar, ciclo do lote e integração com o mercado. Assim, um efeito positivo para CVD significa que domicílios rurais em estágios mais avançados do ciclo de vida (por exemplo, com maior número de adultos ou menor razão de dependência) têm maior chance de apresentar (ou maior área sob) determinada categoria de uso/cobertura do solo. Já um efeito positivo em relação ao CL sugere que domicílios mais antigos (com maior tempo de residência no lote) têm maior chance de apresentar (ou maior área sob) determinada categoria de uso/cobertura do solo. Por fim, um efeito negativo de IM implica que domicílios mais distantes ou com pior acessibilidade aos mercados têm maior chance de apresentar (ou maior área sob) determinada categoria de uso/cobertura do solo. Os efeitos estão apresentados com base no nível de significância estatística reportado nos artigos revisados. ${ }^{2}$

Os resultados sumarizados no Quadro 1 sugerem três pontos centrais:

- a influência do CVD e do CL sobre o uso da terra depende do indicador analisado;

- a influência do CL sobre o uso da terra sobrepõe-se aos indicadores de CVD em fronteiras consolidadas (em especial, Santarém, BR-230, Napo \& Sucumbios);

- a influência dos indicadores de IM prevalece sobre o CVD e o CL.

Em relação ao primeiro ponto, nota-se que os indicadores de CVD explicam meIhor a mudança na cobertura florestal (em especial o desmatamento), ao passo que o $\mathrm{CL}$ tem efeito mais consistente sobre a mudança no uso do solo e sobre os indicadores de capital (renda/bens). A predominância do CVD sobre o CL em relação ao desmatamento é corroborada por estudos empíricos inspirados nos modelos demográficos domiciliares em diversas áreas de assentamento da Amazônia (PAN et al., 2007; CALDAS et al., 2007 e 2003; WALKER, 2003; WALKER; MORAN; ANSELIN, 2000; PICHÓN et al., 1997; JONES et al., 1995). Outras evidências sugerem que o CL é mais influente em relação aos sistemas de uso do solo entre pequenos agricultores (PAN; BILSBORROW, 2005; BARBIERI; BILSBORROW; PAN, 2005; WALKER et al., 2002; PERZ; WALKER, 2002;

\footnotetext{
2 Nos casos em que não há indicação de significância, a técnica utilizada não lida com testes de hipótese. Como exemplo, alguns artigos são baseados em análise de agrupamento ou em técnicas de simulação, as quais não utilizam testes baseados em distribuição de probabilidade.
} 
PERZ, 2001; MARQUETTE, 1998; PICHÓN et al., 1997; MURPHY; BILSBORROW; PICHÓN, 1997; JONES et al., 1995).

O segundo ponto sugere que, quando a fronteira se consolida, o domicílio rural passa a experimentar dois ciclos distintos: um ciclo demográfico domiciliar e um ciclo do lote, independente do primeiro (VANWEY; D'ANTONA; BRONDÍZIO, 2007; BARBIERI; BILSBORROW; PAN, 2005). Esse ciclo do lote é o resultado do aprendizado em relação às características físico-químicas do solo no lote e ao ambiente da fronteira de um modo mais amplo (GUEDES, 2010; SUMMERS, 2008; BARBIERI et al., 2005). Fronteiras mais antigas, por exemplo, tendem a ter um efeito do ciclo do lote mais consistente do que os indicadores do ciclo de vida, em especial em relação ao gerenciamento do uso da terra (VANWEY; D'ANTONA; BRONDÍZIO, 2007; CAVIGLIA-HARRIS; SILLS, 2005; WALKER; MORAN; ANSELIN, 2000). Isso é explicado pelo efeito-tempo e efeito-demonstração, os quais cumprem o papel de reduzir a necessidade de experimentação excessiva com o solo e com as culturas, à medida que se ganha conhecimento sobre o uso ótimo da terra.

Quanto ao envolvimento com o mercado (terceiro ponto), duas variáveis têm sido empregadas nos modelos empíricos: a distância ao centro urbano mais próximo e as condições de acessibilidade em períodos chuvosos. Os resultados indicam (Quadro 1) que domicílios que se encontram mais distantes dos centros urbanos e que possuem piores condições de acesso têm maior superfície contínua de mata intacta, menor área destinada à agricultura comercial e maior chance de adotar técnicas extensivas de manejo (CALDAS et al., 2007 e 2003; MENA et al., 2006; PAN et al., 2004; PERZ, 2003 e 2001; WALKER et al., 2002; GODOY; FRANKS; CLAUDIO, 1998; MARQUETTE, 1998; PICHÓN, 1997; ALSTON; LIBECAP; SCHNEIDER, 1996; BEAUMONT; WALKER, 1996).
De modo geral, os indicadores de integração com o mercado têm recebido maior respaldo empírico significativo do que os indicadores de CVD e CL (terceiro ponto), corroborando o argumento de que, em pós-fronteiras, os pequenos agricultores passam a responder crescente e racionalmente aos estímulos de mercado, amadurecendo e diversificando suas estratégias de sobrevivência. Essas estratégias multidimensionais permitem que estímulos exógenos ao domicílio possam ser efetivamente revertidos em aumento das áreas sob cultivo comercial, uma vez que o domicílio rural aumenta sua atuação espacial (pelas migrações rural-rural e rural-urbana) e soluciona possíveis restrições creditícias por meio de remessas de dinheiro por parte de membros da família com ocupações não-agrícolas (GUEDES, 2010).

\section{Discussão dos resultados}

A grande popularidade dos modelos inspirados na teoria do ciclo de vida domiciliar deveu-se a algumas condições iniciais das fronteiras agrícolas amazônicas que favoreciam sua aplicabilidade: terra abundante; espaço pouco urbanizado; e relativo isolamento das áreas colonizadas em relação aos mercados (CALDAS et al., 2007). O cenário da Amazônia hoje é radicalmente distinto do encontrado no início dos projetos de colonização e assentamento dirigidos dos anos $1970 .{ }^{3}$ Famílias de agricultores cultivando basicamente para subsistência sofreram crescentes influências externas advindas do desenvolvimento das redes viárias, da expansão dos centros urbanos regionais e do desenvolvimento dos mercados locais (PFAFF et al., 2009). Respondendo a esses estímulos externos, os agricultores aumentaram gradualmente suas áreas sob usos comerciais do solo e, ao mesmo tempo, desenvolveram estratégias de diversificação de renda entre os setores da economia, à medida que

\footnotetext{
${ }^{3}$ Embora tenha sofrido uma mudança dramática em termos de ocupação e organização sociodemográfica desde os anos 1970, ainda existem áreas na Amazônia praticamente isoladas da intervenção humana e com características similares às encontradas no início do processo de colonização dirigida.
} 
QUADRO 1

Relações empíricas do CVD, CL e IM (1) sobre uso da terra em fronteiras agrícolas

\begin{tabular}{|c|c|c|c|c|c|}
\hline Fonte & Região Geográfica & Variável Endógena & CVD & CL & IM \\
\hline Almeida (1992) & BR-230 & Renda agropecuária & $(+) n s$ & $(+)^{\star \star}$ & - \\
\hline \multirow[t]{2}{*}{ Jones et al. (1995) } & \multirow[t]{2}{*}{ Rondônia } & Desmatamento & $(+)^{*}$ & $(+) n s$ & $(+)^{*}$ \\
\hline & & Uso comercial & - & $(+)^{*}$ & $(-) \mathrm{ns}$ \\
\hline Almeida e Campari (1995) & BR-230 & Desmatamento & $(-) n s$ & $(+) n s$ & $(+)^{*}$ \\
\hline Alston, Libecap e Schneider (1996) & BR-230 & Uso comercial & $(+) n s$ & $(-) n s$ & $(-)^{* *}$ \\
\hline Beaumont e Walker (1996) & $\mathrm{AB}, \mathrm{AR}, \mathrm{IR}(2)$ & Uso sem pousio & - & - & $(-)$ \\
\hline \multirow{2}{*}{ Thapa, Bilsborrow e Murphy (1996) } & \multirow{2}{*}{ Napo e Sucumbios } & Desmatamento & - & $(+)^{\star \star}$ & - \\
\hline & & Uso comercial & - & $(+)^{\star *}$ & - \\
\hline Murphy, Bilsborrow e Pichón (1997) & Napo e Sucumbios & Renda Total / Bens & $(+) n s$ & $(+)^{\star *}$ & $(-)^{\star \star}$ \\
\hline \multirow[t]{2}{*}{ Pichón (1997) } & \multirow{2}{*}{ Napo e Sucumbios } & Desmatamento & $(+)^{\star \star}$ & $(+)^{\star *}$ & $(-)^{\star \star}$ \\
\hline & & Uso comercial & $(+)^{\star \star}$ & $(+)^{\star \star}$ & $(-)^{\star \star}$ \\
\hline Godoy, Franks e Claudio (1998) & Beni & Uso fertilizante & $(+)^{\star \star}$ & - & $(-)^{\star \star *}$ \\
\hline \multirow[t]{2}{*}{ Marquette (1998) } & \multirow{2}{*}{ Napo e Sucumbios } & Desmatamento & $(+)^{\star \star}$ & $(+) n s$ & $(-)^{\star \star}$ \\
\hline & & Uso comercial & $(+)^{\star \star}$ & $(+) n s$ & $(-)^{\star \star}$ \\
\hline McCracken et al. (1999) & Altamira & Desmatamento & - & $(+)^{*}$ & $(+)^{*}$ \\
\hline \multirow[t]{2}{*}{ Walker, Moran e Anselin (2000) } & \multirow{2}{*}{ Altamira / Uruará } & Desmatamento & $(+)^{*}$ & $(+)^{\star \star}$ & - \\
\hline & & Uso comercial & - & $(-)^{\star \star}$ & - \\
\hline \multirow[t]{2}{*}{ Murphy (2001) } & \multirow{2}{*}{ Napo e Sucumbios } & Renda agropecuária & - & $(+)^{\star \star}$ & $(-)^{\star \star}$ \\
\hline & & Trabalho não-agrícola & $(+)^{\star \star}$ & $(+)^{\star \star}$ & - \\
\hline \multirow[t]{2}{*}{ Perz (2001) } & \multirow{2}{*}{ Uruará } & Uso comercial & $(+)^{\star \star}$ & $(+)^{\star *}$ & $(-) n s$ \\
\hline & & Uso não-comercial & $(-) n s$ & $(-) n s$ & $(-)^{\star *}$ \\
\hline \multirow[t]{2}{*}{ Perz e Walker (2002) } & \multirow{2}{*}{ Uruará } & Pousio & $(+) n s$ & $(+)^{\star \star}$ & $(+) n s$ \\
\hline & & Abandonada & $(-)^{\star \star}$ & $(+) n s$ & $(+) n s$ \\
\hline Walker et al. (2002) & Uruará & Uso comercial & $(+)^{*}$ & $(+)^{\star *}$ & $(-)^{\star \star}$ \\
\hline Caldas et al. (2003) & Uruará & Desmatamento & $(+)^{\star *}$ & $(+) n s$ & $(-)^{\star \star}$ \\
\hline Perz (2003) & Uruará & Tecnologia intensiva & $(+) n s$ & $(-) \mathrm{ns}$ & $(-)^{\star \star}$ \\
\hline Walker (2003) & Uruará & Desmatamento & $(+)$ & - & - \\
\hline \multirow[t]{2}{*}{ Browder, Pedlowski e Summers (2004) } & \multirow{2}{*}{$\begin{array}{l}\text { Nova União, Alto Paraíso e } \\
\text { Rolim de Moura }\end{array}$} & Uso comercial & $(+)^{\star}$ & $(+) n s$ & - \\
\hline & & Uso não-comercial & $(-) n s$ & $(-) n s$ & - \\
\hline Pan et al. (2004) & Napo e Sucumbios & Desmatamento & $(+)$ & $(+)$ & $(-)$ \\
\hline \multirow[t]{2}{*}{ Barbieri, Bilsborrow e Pan (2005) } & \multirow{2}{*}{ Napo e Sucumbios } & Desmatamento & - & $(+)^{\star \star}$ & - \\
\hline & & Uso comercial & - & $(+)^{* \star}$ & - \\
\hline \multirow[t]{3}{*}{ Caviglia-Harris e Sills (2005) } & \multirow{3}{*}{$\begin{array}{l}\text { Tapajós e Ouro Preto } \\
\text { d'Oeste }\end{array}$} & Desmatamento & $(+)^{\star \star}$ & $(+) n s$ & - \\
\hline & & Uso comercial & $(+)^{\star \star}$ & $(+) n s$ & - \\
\hline & & Uso não-comercial & $(-)^{\star \star}$ & $(+) n s$ & - \\
\hline \multirow[t]{3}{*}{ Pan e Bilsborrow (2005) } & & Desmatamento & $(+)^{\star \star}$ & - & $(-)^{\star \star}$ \\
\hline & Napo e Sucumbios & Uso comercial & $(+)^{\star \star}$ & - & $(-) n s$ \\
\hline & & Uso não-comercial & $(-)^{\star \star}$ & - & $(+) n s$ \\
\hline Aldrich et al. (2006) & Uruará & Consolidação & $(+)$ & - & - \\
\hline Mena et al. (2006) & Cuyabeno & Desmatamento & $(+) n s$ & - & $(+) n s$ \\
\hline Caldas et al. (2007) & Uruará & Desmatamento & $(+)^{\star \star}$ & $(+) n s$ & $(-)^{\star \star}$ \\
\hline Pan et al. (2007) & Napo e Sucumbios & Desmatamento & $(+)^{\star \star}$ & $(+)^{\star \star}$ & $(+) n s$ \\
\hline VanWey, D’Antona e Brondízio (2007) & & Desmatamento & $(+) n s$ & - & - \\
\hline & Altamira / Santarém & Uso Comercial & $(+) n s$ & - & - \\
\hline & & Uso não-comercial & $(-)^{*}$ & - & - \\
\hline Barbieri, Carr e Bilsborrow (2009) & Napo e Sucumbios & Migração & $(+)^{\star *}$ & - & $(+)^{\star \star \star}$ \\
\hline
\end{tabular}

Fonte: Elaboração dos autores com base nas fontes citadas.

(1) $C V D=$ Interpretar efeito em relação a domicílios mais velhos. $C L=$ Interpretar efeito em relação a domicílios com maior tempo de residência no lote/região. IM = Interpretar efeito como domicílios mais distantes do mercado/pior acessibilidade.

(2) AB - Amazônia Brasileira; AR = África Rural; IR = Índia Rural.

Nota: ${ }^{*}=$ valor de $p<0,10 ;{ }^{* *}=$ valor de $p<0,05 ; n s=$ não significativo. 
encontravam dificuldades em atender suas necessidades de consumo apenas por meio da produção agrícola (CAVIGLIA-HARRIS; SILLS, 2005; MURPHY, 2001).

As diferentes relações dos produtores rurais com os mercados e suas respostas por meio da migração, da redução de fecundidade e do ajuste no gerenciamento das culturas agrícolas foram se consolidando gradualmente, transformando a Amazônia numa colagem de domicílios em distintas fases de inserção ao ambiente de pós-fronteira (BARBIERI; CARR; BILSBORROW, 2009; RODRIGUES et al., 2009; SUMMERS, 2008). Por essa razão, a mudança nos estágios do ciclo de vida domiciliar foi perdendo poder explicativo, já que outras forças estavam em jogo sobre a organização da produção agrícola (VANWEY; D'ANTONA; BRONDÍZIO, 2007).

A conversão gradual de agricultura de subsistência em agricultura comercial seguiu uma trajetória conjunta à evolução (e reveses) da própria fronteira. Assim, domicílios com maior tempo de residência no lote aprenderam a adaptar suas estratégias de cultivo às características biosíficas da propriedade rural (MORAN et al., 2006). Como grande parte desses pequenos agricultores veio de outras regiões do país, as quais possuem características de organização da produção agrícola e relevos/tipos de solo distintos, a experimentação com o solo nas fronteiras agrícolas amazônicas foi e continua sendo parte importante do processo de consolidação das estratégias de gerenciamento da terra, dando origem a um ciclo do lote, independente da estrutura demográfica domiciliar (VANWEY; D'ANTONA; BRONDÍZIO, 2007; BARBERI; BILSBORROW; PAN, 2005).

VanWey, D’Antona e Brondízio (2007), por exemplo, consideram que, à medida que se consolidam, as estratégias adotadas por antigos agricultores servem como um efeito-demonstração para as práticas de melhor adaptação ao ambiente biofísico, fazendo com que agricultores recém-chegados não necessitem experimentar diferentes técnicas de cultivo antes de encontrar o melhor uso. Os autores chegam a sugerir que o CL, representado pelo tempo de residência do domicílio rural, prevalece sobre o CVD tanto entre domicílios antigos em fronteiras novas quanto em todos os domicílios de fronteiras antigas. Nessas últimas, a consolidação da fronteira já teria cumprido o papel de homogeneizar e difundir o conhecimento das melhores práticas agrícolas.

Outros autores, no entanto, argumentam que essa demonstração depende não somente da cobertura inicial da terra (se desmatada ou não), mas também das redes sociais nas quais o novo proprietário está inserido (BARBIERI; CARR; BILSBORROW, 2009; BRONDÍZIO; MORAN, 2009; FEARNSIDE, 2008). Assim, a abertura de novas áreas (desmatamento) é mais influenciada pela mudança na dinâmica demográfica domiciliar do que o uso do solo, o qual depende diretamente do equacionamento entre características biofísicas, técnicas mais eficientes de produção, capital disponível para investimento e demanda de mercado (GUEDES, 2010).

Summers (2008) argumenta que a influência dos mercados sobre o gerenciamento da terra tende a aumentar com a consolidação das fronteiras, uma vez que essas tornam-se gradativamente mais orientadas para o uso comercial do solo (especialmente perenes e gado). Esse resultado é previsto por modelos teóricos recentes (CALDAS et al., 2007). Com a consolidação das fronteiras, os domicílios rurais diversificam suas estratégias de geração de renda, alocando a mão de obra familiar entre diversos setores da economia (com a expansão dos mercados urbanos locais) e utilizando a migração temporária como eficiente instrumento de minimização de risco (BARBIERI; CARR; BILSBORROW, 2009; MURPHY, 2001). Ao mesmo tempo, a consolidação de diversos lotes em pastos com gado libera mão de obra local, a qual pode ser incorporada por outros lotes que mantêm culturas perenes intensivas em mão de obra, mas que podem sofrer de escassez de trabalho familiar (VANWEY; D'ANTONA; BRONDÍZIO, 2007).

Com as mudanças que ocorreram ao longo do desenvolvimento da fronteira, as relações tradicionais entre demografia e meio ambiente foram sendo postas em questão, uma vez que as estratégias di- 
versificadas das famílias sugerem que os modelos tradicionais de mudança no uso da terra estabeleceram um limite muito rígido em relação ao lote (VANWEY; GUEDES; D'ANTONA, 2008). Influências cada vez mais difusas, vindas de diferentes escalas (locais, regionais e nacionais/internacionais), representam a principal força por trás da transformação da paisagem nas pós-fronteiras da Amazônia.

\section{Considerações finais}

Desde os anos 1970, cresceu o interesse da comunidade científica pelas consequências da penetração humana em fronteiras agrícolas da Amazônia, até então dominadas por florestas primárias. O grande influxo de imigrantes vindo de distintas regiões causou uma elevação repentina nas taxas de desmatamento, especialmente na porção brasileira da Amazônia.

Com as evidências empíricas dos efeitos negativos do desmatamento em relação a possíveis alterações microclimáticas e à biodiversidade, cientistas de diversos campos começaram a analisar o comportamento dos agentes do desmatamento na região. Os primeiros estudos sugeriam que os colonos migrantes adotavam agricultura extensiva, com baixo nível tecnológico, levando a um avanço espacial das áreas desmatadas. Esse ciclo do colono pioneiro, como é chamado na literatura, era factível devido à abundância de terras e à forma de aquisição de títulos de propriedade - por meio da limpeza do lote.

Nesse contexto, representado pela vaga influência do Estado e pelos baixos níveis de integração da agricultura camponesa com os centros urbanos, a mão de obra familiar representava a principal força capaz de modificar o uso da terra. O desmatamento era basicamente destinado ao cultivo de lavouras anuais para subsistência e criação de gado em pequena escala para provimento de fonte proteica aos membros do domicílio.

Vários estudos em escalas macro (Estado, município) sugeriam o efeito devastador dos fluxos migratórios sobre as florestas tropicais da Amazônia. A preocupação era genuína, já que predominava um sistema de corte-e-queimada, o qual aumenta o potencial de incêndios e erosão e reduz rapidamente a fertilidade do solo. Diante dessas evidências, estudos baseados na motivação dos agentes (escala micro - domicílio rural/ lote) passaram a considerar que a mudança na estrutura demográfica domiciliar representava o principal fator que explicava o avanço do desmatamento. Posteriormente, esses estudos associaram a dinâmica demográfica aos modelos de produção domiciliar, sugerindo que as fases do ciclo de vida do domicílio eram caracterizadas, concomitantemente, por fases de sistemas de uso do solo, partindo da subsistência para a integração com os mercados.

A revisão de literatura aqui apresentada sugere que o ciclo de vida domiciliar tem recebido fraco suporte empírico, a despeito de sua ampla utilização como escopo conceitual para explicar a dinâmica do uso da terra. Os estudos trazem evidências de que forças exógenas ao domicílio rural têm crescentemente influenciado a mudança na paisagem da Amazônia rural. Essas forças são basicamente caracterizadas pelo capital específico adquirido na fronteira sobre as características biofísicas do solo, pelas redes sociais e pela influência crescente dos mercados de venda da produção agrícola e de trabalho não-agrícola. Essas forças são resultantes do desenvolvimento da própria fronteira em direção a um cenário complexo, em que as áreas rurais e urbanas tornam-se mais próximas e interdependentes. Os agricultores, anteriormente restritos ao cultivo para subsistência ou produzindo em pequena escala para os mercados locais, estão agora expostos à influência direta do comportamento dos preços e da demanda por produtos agrícolas vinda de diferentes escalas: desde os mercados regionais até demandas internacionais por produtos in natura da Amazônia.

Nesse cenário, a capacidade da relação entre oferta de mão de obra e necessidade de consumo do domicílio em explicar as recentes transformações na paisagem da Amazônia torna-se limitada. Isso lança um desafio para os modelos de uso da terra baseados na influência da dinâmica demográfica: reconhecer e incorporar 
novos elementos que passam a compor a realidade dos ambientes de pós-fronteira e suas consequências sobre a formação

\section{Referências}

ALDRICH, S. P. et al. Land-cover and land-use change in the brazilian Amazon: smallholders, ranchers, and frontier stratification. Economic Geography, v. 82, n. 3, p. 265-288, July 2006.

ALMEIDA, L. O. de. Deforestation and turnover in Amazon colonization. Washington, CD: World Bank, 1992. Mimeografado.

ALMEIDA, A L. O de.; CAMPARI, J. S. Sustainable settlement in the brazilian Amazon. Oxford, UK: Oxford University Press, 1995.

ALSTON, L. J.; LIBECAP, G. D.; SCHNEIDER, $R$. The determinants and impact of property rights: land titles on the brazilian frontier. Cambridge, MA: National Bureau of Economic Research, 1996 (Texto para discussão, 5405).

BARBIERI, A. F.; BILSBORROW, R. E.; PAN, W. K. Farm household lifecycles and land use in the ecuadorian Amazon. Population and Environment, v. 27, n. 1, p. 1-27, Sep. 2005.

BARBIERI, A. F.; CARR, D. L.; BILSBORROW, R. E. Migration within the frontier: the second generation colonization in the ecuadorian Amazon. Population Research and Policy Review, v. 28, n.3, p. 291-320, Jun. 2009.

BEAUMONT, P. M.; WALKER, R. T. Land degradation and property regimes. Ecological Economics, v. 18, n. 1, p. 55-66, Jul. 1996.

BRONDIZIO, E. S.; MORAN, E. F. Human dimensions of climate change: the vulnerability of small farmers in the Amazon. Philosophical Transactions of the Royal Society B, v. 363 , n. 1498 , p. 1803-1809, May 2008.

BROWDER, J. O.; GODFREY, B. J. Rainforest cities: urbanization, development, and de novas instituições que procuram ajustar as estratégias dos domicílios rurais a esse novo contexto.

globalization of the brazilian Amazon. New York: Columbia University Press, cap. 10, p. 312-347, 1997.

BROWDER, J. O.; PEDLOWSKI, M. A.; SUMMERS, P. M. Land use patterns in the brazilian Amazon: comparative farm-level evidence from Rondônia. Human Ecology, v. 32, n. 2, p. 197-224, Apr. 2004.

CALDAS, M. M. et al. Ciclo de vida da família e desmatamento na Amazônia: combinando informações de sensoriamento remoto com dados primários. Revista Brasileira de Economia, Rio de Janeiro, v. 57, n. 4, p. 683-711, out./dez. 2003.

CALDAS, M. M.et al. Theorizing land cover and land use change: the peasant economy of Amazonian deforestation. Annals of the Association of American Geographers, Washington, v. 97, n. 1, p. 86-110, Jan./ Mar. 2007

CAVIGLIA-HARRIS, J. L.; SILLS, E. O. Land use and income diversification: comparing traditional and colonist populations in the Brazilian Amazon. Agricultural Economics, v. 32, n. 3, p. 221-237, Apr. 2005.

FEARNSIDE, P. M. The roles and movements of actors in the deforestation of brazilian Amazonia. Ecology \& Society, v. 13, n. 1, p. 23-45, 2008.

FOX, J. A.; BROWN, D. L. The struggle for accountability: The World Bank, NGOs and grassroots movements. Cambridge, MA: MIT Press, 1988.

GODOY, R.; FRANKS, J. R.; CLAUDIO, M. A. Adoption of modern agricultural technologies by lowland indigenous groups in Bolivia: the role of households, villages, ethnicity, and markets. Human Ecology, v. 26, n. 3, p. 351-369, Sep. 1998.

GUEDES, G. R. Ciclo de vida domiciliar, ciclo do lote e dinâmica do uso da terra na 
Amazônia rural brasileira - um estudo de caso para Altamira, Pará. Tese (Doutorado). Belo Horizonte, Cedeplar/UFMG, 2010.

JONES, D. W. et al. Farming in Rondonia. Resources and Energy Economics, Amsterdam, v. 17, n. 2, p. 155-188, Aug. 1995.

MARQUETTE, C. M. Land use patterns among small farmer settlers in the northeastern ecuadorian Amazon. Human Ecology, v. 26, n. 4, p. 573-598, Dec. 1998.

MCCRACKEN, S. D. et al. Remote sensing and GIS at farm property level: demography and deforestation in the brazilian Amazon. Photogrammetric Engineering \& Remote Sensing, Falls Church, v. 65, n. 11, p. 1311-1320, Nov. 1999.

MENA, C. F. et al. Pressure on the Cuyabeno Wildlife Reserve: development and land use/ cover change in the northern ecuadorian Amazon. World Development, New York, v. 34, n. 10, p. 1831-1849, Oct. 2006.

MORAN, E. F. et al. Human strategies for coping with El Niño related drought in Amazônia. Climatic Change, Dordrecht, v. 77, n. 3/4, p. 343-361, Aug. 2006.

MURPHY, L. L. Colonist farm income, off-farm work, cattle, and differentiation in Ecuador's northern Amazon. Human Organization, v. 60, n. 1, p. 67-79, Apr./Jun. 2001.

MURPHY, L. L.; BILSBORROW, R. E.; PICHÓN, F. J. Poverty and prosperity among migrant settlers in the Amazon rainforest of Ecuador. Journal of Development Studies, v. 34, n. 2, p. 35-66, Dec. 1997.

PAN, W. K. Y. et al. Farm-level models of spatial patterns of land use and land cover dynamics in the ecuadorian Amazon. Agriculture Ecosystems \& Environment, Amsterdam, v. 101, n. 2-3, p.117-134, Feb. 2004.

PAN, W. K. Y. et al. Forest clearing in the ecuadorian Amazon: a study of patterns over space and time. Population and Research Policy Review, Amsterdam, v. 26, n. 5-6, p. 635-659, Dec. 2007.

PAN, W. K. Y.; BILSBORROW, R. E. The use of a multilevel statistical model to analyze factors influencing land use: a study of the ecuadorian Amazon. Global and Planetary Change, v. 47, n. 2-4, p. 232-252, Jul. 2005.

PERZ, S. G. Household demographic factors as life cycle determinants of land use in the Amazon. Population Research and Policy Review, v. 20, n. 3, p. 159-186, Jun. 2001.

PERZ, S. G. Social determinants and land use correlates of agricultural technology adoption in a forest frontier: a case study in the brazilian Amazon. Human Ecology, v. 31, n. 1, p. 133-165, Mar. 2003.

PERZ, S. G.; WALKER, R. T. Household life cycles and secondary forest cover among smallholders in the Amazon. World Development, v. 30, n. 6, p. 1009-1027, Jun. 2002.

PICHÓN, F. J. Colonist land-allocation decisions, land use, and deforestation in the ecuadorian Amazon frontier. Economic Development and Cultural Change, v. 45, n. 4, p. 707-744, Jul. 1997.

PFAFF, A. et al. Road impacts in brazilian Amazonia. In: KELLER, M.; BUSTAMENTE, M.; GASH, J.; DIAS, P. (Eds.). Amazonia and global change. Washington: American Geophysical Union, 2009.

RODRIGUES, A. S. L.; EWERS, R. M.; PARRY, L.; SOUZA JR., C.; VERÍSSIMO, A.; BALMFORD, A. Boom-and-bust development patterns across the Amazon deforestation frontier. Science, v. 324, p. 1435-1437, 2009.

ROTHER, E. T. Revisão sistemática X revisão narrativa. Acta Paul. Enferm., São Paulo, v. 20, n. 2, abr./jun. 2007.

SHERBININ, A. et al. Rural household demographics, livelihoods and the environment. Global Environmental Change, Guildford, v. 18, n. 1, p. 38-53, Feb. 2008.

SIMMONS, C. S.; WALKER, R. T.; ARIMA, E. Y.; ALDRICH, S. P.; CALDAS, M. M. The Amazon land war in the south of Pará. Annals of the Association of American Geographers, v. 97, n. 3, p. 567-592, Sep. 2007.

SUMMERS, P. M. The post-frontier: land use and social change in the brazilian 
Amazon (1992-2002). Tese (Doutorado) Environmental Design and Planning, Virginia Polytechnic Institute and State University, Blacksburg, Virginia, 2008.

THAPA, K. K.; BILSBORROW, R. E.; MURPHY, L. Deforestation, land use, and women's agricultural activities in the ecuadorian Amazon. World Development, v. 24, n. 8, p. 1317-1332, Aug. 1996.

THORNER, D.; KERBLAY, B.; SMITH, R. E. F. (Eds.). Chayanov on the theory of the peasant economy. Homewood, IL: Richard D. Irwin, 1986.

TURNER, F. J. The frontier in american history. New York: Holt, 1920. Disponivel em: <http://xroads.virginia.edu/ Hyper/ TURNER/>. Acesso em: 07 fev. 2009.

VANWEY, L. K.; D’ANTONA, A. O.; BRONDÍZIO, E. S. Household demographic change and land use/land cover change in the brazilian Amazon. Population and Environment, v. 28 , n. 3, p. 163-185, Jan. 2007.

VANWEY, L. K.; GUEDES, G. R.; D'ANTONA, A. O. Land use change in Altamira settlement area, Pará, Brazil: patterns associated with property owner migration or ownership change. In: THE ANNUAL MEETING OF THE POPULATION ASSOCIATION OF AMERICA. [Proceedings...]. Nova Orleans, Silver Spring, MD: PAA, 2008.

WALKER, R. T. Mapping process to pattern in the landscape change of the amazonian frontier. Annals of the Association of American Geographers, v. 93, n. 2, p. 376398, Apr./Jun. 2003.

WALKER, R. T. Theorizing land-cover and land-use change: the case of tropical deforestation. International Regional Science Review, v. 27, n. 3, p. 247-270, Jul. 2004.

WALKER, R. T. et al. Land use and land cover change in forest frontiers: the role of household life cycles. International Regional Science Review, Philadelphia, V. 25, n. 2, p. 169-199, Apr. 2002.

WALKER, R. T.; MORAN, E. F.; ANSELIN, L. Deforestation and cattle ranching in the brazilian Amazon: external capital and household processes. World Development, v. 28 , n. 4, p. 683-699, Apr. 2000.

Recebido para publicação em 08/06/2010 Aceito para publicação em 30/06/2010 